\section{Lungenkrebs: Länger leben dank Betablockern?}

\author{
Es gibt Hinweise, dass Betablocker möglicherweise die Progression von \\ Krebserkrankungen verlangsamen. US-Ärzte überprüften diese Theorie nun \\ bei Patienten mit nichtkleinzelligem Lungenkarzinom (NSCLC).
}

D ie 5-Jahres-Überlebensrate bei Patienten mit nicht operablem NSCLC beträgt trotz Fortschritten in der systemischen und der Radiotherapie derzeit nur etwa $15 \%$. Bei der Ausbildung von Metastasen, der Haupttodesursache beim NSCLC, könnte eine chronische Stressreaktion mit einer verlängerten Katecholamin-Exposition eine Rolle spielen: In präklinischen Studien konnte Noradrenalin über beta-adrenerge Rezeptoren die Migration von Tumorzellen stimulieren. Betablocker sind daher möglicherweise in der Lage, das Fortschreiten der Krebserkrankung aufzuhalten; darauf weisen auch retrospektive Studien bei Brustkrebs- und Melanompatienten hin. In einer neuen Studie wurde bei NSCLC-Patienten ebenfalls ein lebensverlängernder Effekt beobachtet.

Ärzte am M.D. Anderson Cancer Center in Houston, TX/USA, haben retro- spektiv die Daten von 722 Patienten ausgewertet, die sich wegen eines lokal fortgeschrittenen NSCLC einer definitiven Radiotherapie mit einer Gesamtdosis von $60-87,4$ Gy unterzogen hatten. Die Mehrheit der Patienten im Alter zwischen 34 und 95 Jahren hatte ein Stadium-III-Karzinom. 155 Patienten hatten zur Zeit der Bestrahlung einen Betablo-

Letztere überlebten median 23,7 Monate, Patienten ohne Betablocker dagegen nur 18,6 Monate. Wurden Tumorstadium und -histologie, Gesundheitszustand und Therapieregime berücksichtigt, entsprach dies einer Reduktion der Gesamtmortalität um $22 \%$ während eines medianen Follow-up von 44 Monaten. Auch die Überlebenszeit ohne Fernmetastasen und ohne Rezidive war bei den Betablockerpatienten signifikant länger. Nur im Hinblick auf das Überleben ohne lokoregionäre Rezidive becker erhalten. stand kein Unterschied zwischen den beiden Gruppen.

Aus diesem Grund vermuten die $\mathrm{Au}$ toren auch, dass Betablocker „weniger auf den Primärtumor wirken, sondern vielmehr in der Signalkaskade eingreifen, die zur Metastasierung führt“. In der Studie hatten Patienten mit längerem Überleben vor allem beta-1-selektive Betablocker erhalten. Wegen der geringen Patientenzahl könnten aber keine Schlüsse über die Bedeutung von Rezeptorselektivität, Dosis und Applikationsdauer des Betablockers gezogen werden, so Wang und Kollegen. Die Beobachtungen müssten nun zunächst in einer prospektiven Studie überprüft werden.

Fazit: Patienten mit nichtkleinzelligem Lungenkarzinom (NSCLC), die in kurativer Absicht bestrahlt werden, haben eine bessere Prognose, wenn sie gleichzeitig wegen eines Bluthochdrucks oder einer KHK einen Betablocker einnehmen.

Beate Schumacher

Wang $\mathrm{HM}$ et al. Improved survival outcomes with the incidental use of beta-blockers among patients with non-small-cell lung cancer treated with definitive radiation therapy. Ann Oncol. 2013, Jan 8. [Epub ahead of print]

\section{NSCLC: Risikofaktoren für lokoregionäre Rezidive}

\begin{abstract}
Patienten mit nichtkleinzelligem Lungenkarzinom (NSCLC) in den frühen Stadien haben nach Resektion des Tumors relativ gute Überlebenschancen. Welche Faktoren hier speziell das lokoregionäre Rezidivrisiko erhöhen, prüfte ein Autorenteam des renommierten MD Anderson Cancer Center in Texas.
\end{abstract}

$\mathrm{R}^{\mathrm{s}}$ etrospektiv analysierten sie die Daten von 1.402 Patienten, die sich einer Resektion ihres NSCLC unterzogen hatten. Das mediane Alter betrug 67 Jahre. Kein Studienteilnehmer hatte Fernmetastasen oder einen Befall kontralateraler Lymphknotenstationen (Stadien I-III, N0-N1). $70 \%$ der Tumoren waren in den Stadien IA und IB, $84 \%$ ohne Lymphknotenbefall. Die mediane Tumorgröße lag bei $2,7 \mathrm{~cm}$. In $80 \%$ der Fälle führten die Chirurgen eine Lobektomie durch.

Nach einer medianen Beobachtungszeit von 42 Monaten hatten 123 Patienten (9\%) lokoregionäre Rezidive entwickelt. Dabei handelte es sich um 39 Lo- kalrezidive, 59 regionale Rezidive und 25 Fälle mit lokalem plus regionalem Befall. Fernrezidive waren bei 220 Patienten aufgetreten.

In der multivariaten Analyse zeigten sich drei signifikante unabhängige Risikofaktoren für lokale Rezidive: Nach weniger ausgedehnten Operationen (Keiloder Segmentresektionen) traten häufiger Rezidive auf als nach Lobektomie oder Pneumonektomie (6,5 vs. $4 \%$ ). Bei einer Tumorgröße von $>2,7 \mathrm{~cm}$ wurden häufiger Rezidive beobachtet als bei kleineren Tumoren (6 vs. $3 \%$ ). Der Befall der viszeralen Pleura verdoppelte die Rezidivhäufigkeit (8vs. $4 \%$ ). Für regionale Rezidive ergaben sich ebenfalls drei signifikante Faktoren: Lymphknotenbefall (Rezidivraten: $9 \%$ bei N1 vs. $5 \%$ bei N0), Befall der viszeralen Pleura (9\% mit vs. $5 \%$ ohne Befall) sowie lymphovaskuläre Invasion (10\% mit vs. $5 \%$ ohne Invasion).

Fazit: Bestimmte Operationsverfahren sowie Faktoren wie Tumorgröße, Befall der viszeralen Pleura oder lymphovaskuläre Invasion erhöhten die Wahrscheinlichkeit für lokoregionäre Rezidive. Laut den Autoren bestehe die Möglichkeit, betroffene Patienten einer aggressiveren Therapie zuzuführen. Kritisch anzumerken ist, dass lokoregionäre Rezidive deutlich seltener als Fernrezidive auftraten und der Fokus daher mehr auf deren $\mathrm{Ri}$ sikofaktoren liegen sollte. Andreas Fischer

Lopez Guerra JL et al. Risk factors for local and regional recurrence in patients with resected NO-N1 non-small-cell lung cancer, with implications for patient selections for adjuvant therapy. Ann Oncol. 2013;24(1):67-74. 